\title{
On Noncentral Bell Numbers and Their Hankel Transforms
}

\author{
Roberto B. Corcino*, Harren Jaylo-Campos, Amila P. Macodi-Ringia \\ Department of Mathematics, Mindanao State University, Marawi City, Philippines \\ *Corresponding author: rcorcino@yahoo.com
}

Received February 11, 2014; Revised March 17, 2014; Accepted March 26, 2014

\begin{abstract}
The noncentral Stirling numbers of the first and second kind are certain generalization of the classical Stirling numbers of both kinds. In this paper, a kind of generalized Bell numbers, called noncentral Bell numbers, are defined in terms of noncentral Stirling numbers of the second kind. Some properties parallel to the ordinary Bell numbers are established including the Hankel transform of noncentral Bell numbers. Moreover, an alternative proof for the Hankel transform of $(r, \beta)$-Bell numbers is presented.
\end{abstract}

Keywords: Stirling numbers, Bell numbers, Whitney numbers, Dowling numbers, Catalan numbers, binomial transform, Hankel transform

Cite This Article: Roberto B. Corcino, Harren Jaylo-Campos, and Amila P. Macodi-Ringia, "On Noncentral Bell Numbers and Their Hankel Transforms." Turkish Journal of Analysis and Number Theory, vol. 2, no. 2 (2014): 29-36. doi: 10.12691/tjant-2-2-1.

\section{Introduction}

The theory of Hankel matrices has been previously studied by some mathematician and its connections in some areas of mathematics, physics and computer science (see, the works of Desainte-Catherine and Viennot [9], Garcia-Armas and Sethuraman [11], Tamm [22], Vein and Dale [23]). Though, Hankel determinants had been previously studied (see, for example, Aigner [1], Radoux [19], Ehrenborg [10]), the term Hankel Transform was first introduced in Sloane's sequence $A 055878$ [20] and first studied by Layman [15]. Layman used the notion of binomial transform $\left(b_{n}\right)$ of a sequence $\left(a_{n}\right)$ given by

$$
b_{n}=\sum_{k=0}^{n}\left(\begin{array}{l}
n \\
k
\end{array}\right) a_{k}
$$

and the invert transform

$$
a_{n}=\sum_{k=0}^{n}\left(\begin{array}{l}
n \\
k
\end{array}\right)(-1)^{n-k} b_{k},
$$

in establishing some properties of the Hankel transform including the theorem which states that any integer sequence has the same Hankel transform as its binomial or invert transform.

\begin{tabular}{|c|c|}
\hline Hankel transform & Sequences \\
\hline$\{1,1,2,12,288, \ldots\}$ & $\frac{A 000085}{A 005} \underline{A 000110}, \underline{A 000296}, \underline{A 005425}$, \\
\hline$\{1,1,4,144$, & $\underline{A 000142}, \underline{A 000166}, \underline{A 045379}$ \\
$82944, \ldots\}$ & $\underline{A 0103703}, \underline{A 010842}, \underline{A 052186,}, \underline{A 053486}$, \\
\hline
\end{tabular}

Layman found out that some sequences have the same Hankel transform. For instance, the sequence of Catalan numbers $\{1,1,2,3,14,42, \ldots\}$ (sequence $A 000108$ in the EIS) and approximately twenty sequences have the same Hankel transform $\{1,1,1, \ldots\}$. The following are some of the sequences with the same Hankel trans- form.

Also, Layman and Michael Somos found ten sequences $(\underline{\mathrm{A} 055209})$ in the EIS whose Hankel transform is

$$
\left\{\prod_{i=0}^{n}(i !)^{2}\right\},
$$

which was shown theoretically by Radoux [19] to be the Hankel transform of the derangements, or rencontres numbers (A000166).

Several later studies of Hankel transform of some integer sequences were estab- lished. Among them were:

1. Cvetcoć et al. [8], who established the Hankel transform of the sequence of the sum of two adjacent Catalan numbers. More precisely, if we let $a_{n}=$ $C_{n}+C_{n+1}$ where $C_{n}$ is the nth Catalan number, then the Hankel transform of an is

$$
H\left(a_{n}\right)=\left\{F_{2 n+1}\right\}_{n \in N_{o}}
$$

where $F_{n}$ is $n$th Fibonnaci number.

2. Armas and Sethuraman [11], who established the Hankel transform of central binomial coeffficients which is stated as follows:

The zeroth Hankel transform $d_{n}^{(0)}$ of the sequence $\left(\begin{array}{l}2 l \\ l\end{array}\right), \quad l=0,1,2, \ldots \quad$ is the sequence $2^{n-1}$, $n=1,2, \ldots$, and the first Hankel transform $d_{n}^{(1)}$ is the sequence $2^{n}, n=1,2, \ldots$, 
3. Spivey and Steil [21], French (2007), Chamberland and French (2007), Rajković, Ivković and Barry (2007), who established the $k$-binomial transform and Han- kel transform, transformation in preserving the Hankel transform, generalized Catalan numbers and Hankel transformations, and the Hankel transform of the sum of consecutive generalized Catalan numbers, respectively.

4. Aigner [1], who established a characterization of the sequence of Bell numbers $\left(B_{n}\right)$ and proved that this sequence has the Hankel transform which is given by

$$
n ! !=\prod_{k=0}^{n} k !
$$

5. Mezo [16], who recently proved that the Bell numbers and $r$-Bell numbers [18] have the same Hankel transform.

In this present study, certain generalization of Bell numbers which is defined as the sum of noncentral Stirling numbers of the second by M. Koutras [14], will be established. It will also be shown that these generalized Bell numbers has the same Hankel transform as that of the sequence of ordinary Bell numbers.

\section{The Noncentral Bell Numbers}

In 1982, M. Koutras [14] introduced the noncentral Stirling numbers of first and second kind. These numbers denoted by $s_{a}(n, k)$ and $S_{a}(n, k)$ are defined as the coefficients of the following expansions, with parameter $a$,

$$
\begin{aligned}
& (t)_{n}=\sum_{k=0}^{n} s_{a}(n, k)(t-a)^{k} \\
& (t-a)^{n}=\sum_{k=0}^{n} S_{a}(n, k)(t)^{k}
\end{aligned}
$$

where $\quad s_{a}(0,0)=S_{a}(0,0)=1, \quad s_{a}(n, 0)=(a)_{n}$, $S_{a}(n, 0)=(-a)^{n}$ and $s_{a}(0, k)=S_{a}(0, k)=0, n, k \neq 0$.

The following theorems contain some combinatorial identities of the noncentral Stirling numbers of both kind which are established by Koutras [14].

Theorem 2.1. The noncentral Stirling numbers of the first and second kind satisfy the recurrence relations

$$
\begin{gathered}
s_{a}(n+1, k)=s_{a}(n, k-1)+(a-n) s_{a}(n, k) \\
S_{a}(n+1, k)=S_{a}(n, k-1)+(k-a) S_{a}(n, k)
\end{gathered}
$$

where $s_{a}(0,0)=S_{a}(0,0)=1, \quad s_{a}(n, n)=S_{a}(n, n)=1$, and $s_{a}(n, k)=S_{a}(n, k)=0$ if $n, k<0$ or $k>n$.

Note that if $a=0$ in (3) and (4), then we have

$$
\begin{gathered}
s(n+1, k)=s_{a}(n, k-1)-n s(n, k) \\
S(n+1, k)=S_{a}(n, k-1)+k S(n, k) .
\end{gathered}
$$

Thus, the ordinary Stirling numbers can be expressed as

$$
s(n, k)=s_{0}(n, k) \text { and } S(n, k)=S_{0}(n, k) .
$$

Theorem 2.2. The numbers $s_{a}(n, k)$ and $S_{a}(n, k)$ have the following exponential generating functions

$$
\begin{gathered}
f_{k}(u)=\sum_{n=k}^{\infty} s_{a}(n, k) \frac{u^{n}}{n !}=(1+u)^{a}[\log (1+u)]^{k} \\
h_{k}(u)=\sum_{n=k}^{\infty} S_{a}(n, k) \frac{u^{n}}{n !}=e^{-a u} \frac{1}{k !}\left[e^{u}-1\right]^{k}
\end{gathered}
$$

Theorem 2.3. The numbers $s_{a}(n, k)$ and $S_{a}(n, k) S_{a}(n$; k) have the following explicit formula

$$
\begin{gathered}
s_{a}(n, k)=\frac{n !}{k !} \sum_{l=k}^{n}(-1)^{l-k}\left(\begin{array}{c}
a \\
n-l
\end{array}\right) \sum \frac{1}{l_{1} l_{2} \ldots l_{k}} \\
S_{a}(n, k)=\frac{1}{k !} \sum_{l=0}^{k}(-1)^{k-l}\left(\begin{array}{l}
k \\
l
\end{array}\right)(l-a)^{n}
\end{gathered}
$$

Now, let us define the noncentral Bell numbers parallel to the definition of the ordinary Bell numbers.

Definition 2.4. The noncentral Bell numbers, denoted by $B_{a}(n)$, are defined by

$$
B_{a}(n)=\sum_{k=0}^{n} S_{a}(n, k)
$$

In particular, $B_{0}(n)=B(n)$, the ordinary Bell numbers.

Using the exponential generating function of the noncentral Stirling numbers of the second kind, we can obtain the following exponential generating function for $\mathrm{B}_{\mathrm{a}}(\mathrm{n})$.

Theorem 2.5. The noncentral Bell numbers have the following generating functions

$$
\sum_{n=0}^{\infty} B_{a}(n) \frac{u^{n}}{n !}=e^{\left(e^{u}-1\right)-a u}, B_{0}(n)=B(n)
$$

Proof. By making use of the exponential generating function (6) of $S_{a}(n, k)$ we have,

$$
\begin{aligned}
\sum_{n=0}^{\infty} B_{a}(n) \frac{u^{n}}{n !} & =\sum_{n=0}^{\infty}\left(\sum_{k=0}^{n} S_{a}(n, k)\right) \frac{u^{n}}{n !} \\
& =e^{-a u} \sum_{k=0}^{\infty} \frac{1}{k !}\left(e^{u}-1\right)^{k} \\
& =e^{-a u} \cdot e^{e^{u}-1}=e^{\left(e^{u}-1\right)-a u}
\end{aligned}
$$

Hence, the exponential generating function of $B_{a}(n)$ is $e^{\left(e^{u}-1\right)-a u}$.

If $a=0,(9)$ becomes

$$
\sum_{n=0}^{\infty} B_{a}(n) \frac{u^{n}}{n !}=e^{\left(e^{u-1}\right)}
$$

the exponential generating function of the ordinary Bell numbers.

The next theorem contains a kind of Dobinski formula for $B_{a}(n)$.

Theorem 2.6. The noncentral Bell numbers $B_{a}(n)$ can be written in the form of a convergent series

$$
B_{a}(n)=\frac{1}{e} \sum_{k=0}^{\infty} \frac{(k-a)^{n}}{n !}
$$


Proof. Applying the exponential generating function of $B_{a}(n)$ in (9),

$$
\begin{aligned}
\sum_{n=0}^{\infty} B_{a}(n) \frac{u^{n}}{n !} & =e^{\left(e^{u}-1\right)-a u} \\
& =e^{-1} \cdot e^{-a u} \sum_{k=0}^{n} \frac{\left(e^{u}\right)^{k}}{k !} \\
& =e^{-1} \sum_{k=0}^{n} \frac{e^{(k-a) u}}{k !} \\
& =\sum_{n=0}^{\infty}\left[\frac{1}{e} \sum_{k=0}^{n} \frac{(k-a)^{n}}{k !}\right] \frac{u^{n}}{n !}
\end{aligned}
$$

Comparing the coefficient of $\frac{u^{n}}{n !}$, we obtain

$$
B_{a}(n)=\frac{1}{e} \sum_{k=0}^{n} \frac{(k-a)^{n}}{k !} .
$$

The following theorem contains some relations which are useful in establishing the alternative proof of the claim that the sequence of $B_{a}(n)$ has the same Hankel transform as that of the sequence of $B(n)$. This is a kind of a recurrence relation of $B_{a}(n)$.

Theorem 2.7. The noncentral Bell numbers satisfy the relations

$$
\begin{gathered}
B_{a}(n)=\sum_{k=0}^{n}\left(\begin{array}{l}
n \\
k
\end{array}\right) B_{a+1}(k) \\
B_{a+1}(n)=\sum_{k=0}^{n}\left(\begin{array}{l}
n \\
k
\end{array}\right)(-1)^{n-k} B_{a}(k)
\end{gathered}
$$

Proof. Multiplying $e^{u}$ to both side of (9) with a being replaced with $a+1$, we have

$$
\begin{gathered}
e^{u} \sum_{n=0}^{\infty} B_{a+1}(n) \frac{u^{n}}{n !}=e^{\left(e^{u}-1\right)-(a+1) u} \cdot e^{u} \\
\sum_{n=0}^{\infty} \frac{u^{n}}{n !} \sum_{n=0}^{\infty} B_{a+1}(n) \frac{u^{n}}{n !}=e^{\left(e^{u}-1\right)} \cdot e^{-a u} \cdot e^{-u} \cdot e^{u} \\
\sum_{n=0}^{\infty}\left[\sum_{k=0}^{n} \frac{u^{n-k}}{(n-k) !} B_{a+1}(k) \frac{u^{k}}{k !}\right]=e^{\left(e^{u}-1\right)-a u} \\
\sum_{n=0}^{\infty}\left[\sum_{k=0}^{n}\left(\begin{array}{l}
n \\
k
\end{array}\right) B_{a+1}(k) \frac{u^{(n-k)}}{n !}\right]=\sum_{n=0}^{\infty} B_{a}(n) \frac{u^{n}}{n !} \\
\sum_{n=0}^{\infty}\left[\sum_{k=0}^{n}\left(\begin{array}{l}
n \\
k
\end{array}\right) B_{a+1}(k)\right] \frac{u^{n}}{n !}=\sum_{n=0}^{\infty} B_{a}(n) \frac{u^{n}}{n !}
\end{gathered}
$$

Comparing the coefficient of $\frac{u^{n}}{n !}$, we obtain the following relation

$$
B_{a}(n)=\sum_{k=0}^{n}\left(\begin{array}{l}
n \\
k
\end{array}\right) B_{a+1}(k)
$$

Similarly, multiplying $e^{-u}$ to both side of (9), we have

$$
\begin{gathered}
e^{-u} \sum_{n=0}^{\infty} B_{a}(n) \frac{u^{n}}{n !}=e^{\left(e^{u}-1\right)-a u} \cdot e^{-u} \\
\sum_{n=0}^{\infty}(-1)^{n} \frac{u^{n}}{n !} \sum_{n=0}^{\infty} B_{a}(n) \frac{u^{n}}{n !}=e^{\left(e^{u}-1\right)} \cdot e^{-(a+1) u} \\
\sum_{n=0}^{\infty}\left[\sum_{k=0}^{n}(-1)^{n-k} \frac{u^{n-k}}{(n-k) !} B_{a}(k) \frac{u^{k}}{k !}\right]=e^{\left(e^{u}-1\right)-(a+1) u} \\
\sum_{n=0}^{\infty}\left[\sum_{k=0}^{n}\left(\begin{array}{l}
n \\
k
\end{array}\right)(-1)^{n-k} B_{a}(k) \frac{u^{(n-k)} u^{k}}{n !}\right]=\sum_{n=0}^{\infty} B_{a+1}(n) \frac{u^{n}}{n !} \\
\sum_{n=0}^{\infty}\left[\sum_{k=0}^{n}\left(\begin{array}{l}
n \\
k
\end{array}\right)(-1)^{n-k} B_{a}(k)\right] \frac{u^{n}}{n !}=\sum_{n=0}^{\infty} B_{a+1}(n) \frac{u^{n}}{n !}
\end{gathered}
$$

Comparing the coefficient of $\frac{u^{n}}{n !}$,

$$
B_{a+1}(n)=\sum_{k=0}^{n}\left(\begin{array}{l}
n \\
k
\end{array}\right)(-1)^{n-k} B_{a}(k) \text {. }
$$

Remark 2.8. Theorem 4.1.4 implies that $B_{a+1}(n)$ is the binomial transform of $B_{a}(n)$ or $B_{a}(n)$ is the inverse transform of $B_{a+1}(n)$.

\section{The Hankel Transform of Noncentral Bell Numbers}

Let $\mathcal{A}=\left(a_{n, k}\right)$ be the in_nite lower triangular matrix defined recursively by,

$$
\begin{aligned}
a_{n, k}= & a_{n-1, k-1}+[(k+1)-a] a_{n-1, k} \\
& +(k+1) a_{n-1, k+1},(n \geq 1)
\end{aligned}
$$

with the intial condition $a_{0,0}=1, a_{n, k}=0(n<k)$.

The following lemma contains the exponential generating function of the kth column entries of $\mathcal{A}$.

Lemma 3.1. Let $\Psi_{k}(x)$ be the exponential generating function of the kth column of $a_{n, k}$

$$
\Psi_{k}(x)=\sum_{n=0}^{\infty} a_{n, k} \frac{x^{n}}{n !}
$$

then

$$
\Psi_{k}(x)=e^{\left(e^{x}-1\right)-a u} \frac{\left(e^{x}-1\right)^{k}}{k !},(k \geq 0) .
$$

where $\Psi_{0}(x)=\sum_{n=0}^{\infty} B_{a}(x) \frac{x^{n}}{n !}$. That is, the 0-column entries of $\mathcal{A}$ are $B_{a}(n), n=0,1,2, \ldots$.

Proof. By making use of the recurrence relation in (12) we obtain 


$$
\begin{aligned}
& \sum_{n=1}^{\infty} a_{n, k} \frac{x^{n-1}}{(n-1) !} \\
& =\sum_{n=1}^{\infty} a_{n-1, k-1} \frac{x^{n-1}}{(n-1) !}+[(k+1)-a] \sum_{n=1}^{\infty} a_{n-1, k} \frac{x^{n-1}}{(n-1) !} \\
& +(k+1) \sum_{n=1}^{\infty} a_{n-1, k+1} \frac{x^{n-1}}{(n-1) !} \\
& \Psi_{k}^{\prime}(x)=\Psi_{k-1}(x)+[(k+1)-a] \Psi_{k}(x) \\
& +(k+1) \Psi_{k+1}(x)
\end{aligned}
$$

With $\Psi_{k}(x)=e^{u} \frac{\left(e^{x}-1\right)^{k}}{k !}, u=\left(e^{x}-1\right)-a x$, the lefthand side (LHS) of (13) yields

$$
\begin{aligned}
\Psi_{k}^{\prime}(x)= & e^{\left(e^{x}-1\right)-a x} \frac{\left(e^{x}-1\right)^{k-1}}{(k-1) !} \cdot e^{x} \\
& +e^{\left(e^{x}-1\right)-a x} \frac{\left(e^{x}-1\right)^{k}}{k !} \cdot\left(e^{x}-a\right) \\
\Psi_{k}^{\prime}(x)= & e^{\left(e^{x}-1\right)-a x} \frac{\left(e^{x}-1\right)^{k-1}}{(k-1) !} \cdot e^{x} \\
& +e^{\left(e^{x}-1\right)-a x} \frac{\left(e^{x}-1\right)^{k}}{k !} \cdot e^{x} \\
& -a e^{\left(e^{x}-1\right)-a x} \frac{\left(e^{x}-1\right)^{k}}{k !} .
\end{aligned}
$$

On the other hand, the right-hand side (RHS) of (13) gives

$$
\begin{aligned}
R H S= & e^{u} \frac{\left(e^{x}-1\right)^{k-1}}{(k-1) !}+[(k+1)-a] e^{u} \frac{\left(e^{x}-1\right)^{k}}{k !} \\
& +(k+1) e^{u} \frac{\left(e^{x}-1\right)^{k+1}}{(k+1) !} \\
= & e^{u} \frac{\left(e^{x}-1\right)^{k-1}}{(k-1) !}+k e^{u} \frac{\left(e^{x}-1\right)^{k}}{k !}+e^{u} \frac{\left(e^{x}-1\right)^{k}}{k !} \\
& -a e^{u} \frac{\left(e^{x}-1\right)^{k}}{k !}+e^{u} \frac{\left(e^{x}-1\right)^{k+1}}{k !} \\
= & e^{u} \frac{\left(e^{x}-1\right)^{k-1}}{(k-1) !}+e^{u} \frac{\left(e^{x}-1\right)^{k-1}}{(k-1) !}\left(e^{x}-1\right)+e^{u} \frac{\left(e^{x}-1\right)^{k}}{k !} \\
& -a e^{u} \frac{\left(e^{x}-1\right)^{k}}{k !}+e^{u} \frac{\left(e^{x}-1\right)^{k}}{k !}\left(e^{x}-1\right) \\
= & e^{u} \frac{\left(e^{x}-1\right)^{k-1}}{(k-1) !}+e^{u} \frac{\left(e^{x}-1\right)^{k-1}}{(k-1) !} e^{x} \\
& -e^{u} \frac{\left(e^{x}-1\right)^{k-1}}{(k-1) !}+e^{u} \frac{\left(e^{x}-1\right)^{k}}{k !}-a e^{u} \frac{\left(e^{x}-1\right)^{k}}{k !} \\
& +e^{u} \frac{\left(e^{x}-1\right)^{k}}{k !} e^{x}-e^{u} \frac{\left(e^{x}-1\right)^{k}}{k !}
\end{aligned}
$$

$$
\begin{aligned}
& =e^{u} \frac{\left(e^{x}-1\right)^{k-1}}{(k-1) !} e^{x}-a e^{u} \frac{\left(e^{x}-1\right)^{k}}{k !}+e^{u} \frac{\left(e^{x}-1\right)^{k}}{k !} e^{x} \\
& =e^{u} \frac{\left(e^{x}-1\right)^{k-1}}{(k-1) !} e^{x}+e^{u} \frac{\left(e^{x}-1\right)^{k}}{k !} e^{x}-a e^{u} \frac{\left(e^{x}-1\right)^{k}}{k !} \\
& =e^{\left(e^{x}-1\right)-a x} \frac{\left(e^{x}-1\right)^{k-1}}{(k-1) !} e^{x} \\
& +e^{\left(e^{x}-1\right)-a x} \frac{\left(e^{x}-1\right)^{k}}{k !}\left(e^{x}-a\right)=\Psi_{k}^{\prime}(x)
\end{aligned}
$$

This implies that the generating function $\Psi_{k}(x)=e^{\left(e^{x}-1\right)-a x} \frac{\left(e^{x}-1\right)^{k}}{k !},(k \geq 0)$ is a unique solution to the differential equation (13). Thus, the exponential generating function of the kth column of $\mathcal{A}$ is given by

$$
\Psi_{k}(x)=e^{\left(e^{x}-1\right)-a x} \frac{\left(e^{x}-1\right)^{k}}{k !} .
$$

Lemma 3.2. Let be the nth row of $\mathcal{A}$. Define $r_{n} \circ r_{l}=\sum_{k \geq 0} a_{n, k} a_{l, k} k !$ then $r_{n} \circ r_{l}=a_{n+l, 0}=B_{a}(n+1)$ for all $n$ and $l$.

Proof. We prove this by induction on $n$. If $n=0$ we have $r_{0} \circ r_{l}=\sum_{k \geq 0} a_{0, k} a_{l, k} k ! . \quad$ Since $\quad a_{0, k}=0 \quad \forall k \geq 0$, $r_{0} \circ r_{l}=a_{0,0} a_{l, 0} 0 !=a_{0+l, 0} \quad \forall l . \quad$ Suppose that $r_{m} \circ r_{l}=a_{m+l, 0}$ Suppose that $m \leq n-1$ and all $l$. Then by (12) and intechanging the summation we have,

$$
\begin{aligned}
& r_{n} \circ r_{l}=\sum_{k \geq 0} a_{n, k} a_{l, k} k ! \\
& =\sum_{k \geq 0}\left(\begin{array}{l}
a_{n-1, k}+[(k+1)-a] a_{n-1, k} \\
+(k+1) a_{n-1, k+1}
\end{array}\right) a_{l, k} k ! \\
& =\sum_{k \geq 0} a_{n-1, k-1} a_{l, k} k \text { ! } \\
& +\sum_{k \geq 0}[(k+1)-a] a_{n-1, k} a_{l, k} k ! \\
& +\sum_{k \geq 0}(k+1) a_{n-1, k+1} a_{l, k} k ! \\
& =\sum_{k \geq 0} a_{n-1, k-1} a_{l, k} k \text { ! } \\
& +\sum_{k \geq 0} a_{n-1, k} a_{l, k}[(k+1)-a] k ! \\
& +\sum_{k \geq 0} a_{n-1, k+1} a_{l, k}(k+1) \text { ! } \\
& =\sum_{k \geq-1} a_{l, k+1} a_{n-1,(k+1)-1}(k+1) \text { ! } \\
& +\sum_{k \geq 0} a_{l, k} a_{n-1, k}[(k+1)-a] k ! \\
& +\sum_{k \geq-1} a_{l, k-1} a_{n-1,(k-1)+1}[(k-1)+1] \text { ! } \\
& =\sum_{k \geq 0}\left(\begin{array}{c}
a_{l, k+1} a_{n-1, k}(k+1) ! \\
+a_{l, k} a_{n-1, k}[(k+1)-a] k !+a_{l, k-1}
\end{array}\right) a_{n-1, k} k !
\end{aligned}
$$




$$
\begin{aligned}
& =\sum_{k \geq 0}\left(a_{l, k-1}+[(k+1)-a] a_{l, k}+a_{l, k+1}\right) a_{n-1, k} k ! \\
& =\sum_{k \geq 0} a_{n-1, k} a_{l+1, k} k !
\end{aligned}
$$

By the inductive hypothesis,

$$
r_{n} \circ r_{l}=a_{(n-1)+(l+1), 0}=a_{n+l, 0}=(B(n+l)) .
$$

We are now ready to introduce the following theorem.

Theorem 3.3. The noncentral Bell numbers have the Hankel transform

$$
\left|\begin{array}{ccccc}
B_{a}(0) & B_{a}(1) & B_{a}(2) & \ldots & B_{a}(n) \\
B_{a}(1) & B_{a}(2) & B_{a}(3) & \cdots & B_{a}(n+1) \\
\vdots & \vdots & \vdots & \cdots & \vdots \\
B_{a}(n) & B_{a}(n+1) & B_{a}(n+2) & \cdots & B_{a}(2 n)
\end{array}\right|=\prod_{j=0}^{n} j !
$$

Proof. Let $\mathcal{A}_{n}$ be the submatrix of $\mathcal{A}$ consisting of the rows and columns numbered 0 to $\mathrm{n}$. Clearly, $\operatorname{det} \mathcal{A}_{n}=1$, since $\mathcal{A}_{n}$ is a lower triangular matrix with diagonal 1 . It follows that $\operatorname{det} \mathcal{A}_{n}^{T}=1$. Let $\overline{\mathcal{A}}_{n}=\left(j ! a_{i, j}\right)_{0 \leq i, j \leq n}$. Then

$$
\operatorname{det} \overline{\mathcal{A}}_{n}=\prod_{j=0}^{n} j !
$$

By Lemma 3.2,

$$
\overline{\mathcal{A}}_{n} \mathcal{A}_{n}^{T}=\left(a_{i, j}^{\prime}\right)_{0 \leq i, j \leq n}
$$

where

$$
a_{i, j}^{\prime}=\sum_{k=0}^{n} k ! a_{i, k} a_{j, k}=a_{i+j, 0}=B_{a}(i+j) .
$$

That is,

$$
\overline{\mathcal{A}}_{n} \mathcal{A}_{n}^{T}=\left(B_{a}(i+j)\right)_{0 \leq i, j \leq n}
$$

Thus,

$$
\operatorname{det}\left(\overline{\mathcal{A}}_{n} \mathcal{A}_{n}^{T}\right)=\left(\operatorname{det} \overline{\mathcal{A}}_{n}\right)\left(\operatorname{det} \mathcal{A}_{n}^{T}\right)=1 \cdot \prod_{j=0}^{n} j !=\prod_{j=0}^{n} j !
$$

The theorem can also be proved without using Lemma 3.2. One can use the fact that $B_{0}(n)=B(n)$, the ordinary Bell numbers. This means that

$$
\operatorname{det}\left(B_{0}(n)\right)=\prod_{j=0}^{n} j !
$$

That is, the Hankel transform of $\left(B_{0}(n)\right)$ is $(0 ! 1 ! 2 ! 3 ! \ldots)$. By Theorem 2.7, $B_{1}(n)$ is the binomial transform of $B_{0}(n)$ and so, by Layman's theorem, $B_{0}(n)$ and $B_{1}(n)$. have the same Hankel transform. Again by Theorem 2.7, $B_{1}(n)=\sum_{k=0}^{n}\left(\begin{array}{l}n \\ k\end{array}\right) B_{2}(k)$ implies that $B_{2}(n)$ is the binomial transform of $B_{1}(n)$. So, by Layman's
Theorem, $B_{1}(n)$ and $B_{2}(n)$ have the same Hankel transform. Continuing this process and again, since

$$
B_{a}(n)=\sum_{k=0}^{n}\left(\begin{array}{l}
n \\
k
\end{array}\right) B_{a+1}(k),
$$

by induction, $B_{a}(n)$ and $B_{a+1}(n)$ have the same Hankel transform. Hence, $B_{0}(n)$ and $B_{a}(n)$ have the same Hankel transform. Thus,

$$
\begin{aligned}
& \left|\begin{array}{ccccc}
B_{a}(0) & B_{a}(1) & B_{a}(2) & \ldots & B_{a}(n) \\
B_{a}(1) & B_{a}(2) & B_{a}(3) & \cdots & B_{a}(n+1) \\
\vdots & \vdots & \vdots & \cdots & \vdots \\
B_{a}(n) & B_{a}(n+1) & B_{a}(n+2) & \cdots & B_{a}(2 n)
\end{array}\right| \\
& =\left|\begin{array}{ccccc}
B_{0}(0) & B_{0}(1) & B_{0}(2) & \ldots & B_{0}(n) \\
B_{0}(1) & B_{0}(2) & B_{0}(3) & \cdots & B_{0}(n+1) \\
\vdots & \vdots & \vdots & \cdots & \vdots \\
B_{0}(n) & B_{0}(n+1) & B_{0}(n+2) & \cdots & B_{0}(2 n)
\end{array}\right|=\prod_{j=0}^{n} j !
\end{aligned}
$$

For possible future research, it is interesting to establish q-analogues of the noncentral Stirling and Bell numbers and to determine their Hankel transforms. It will be more interesting if one can establish connections with those qanalogues of Stirling and Bell numbers via normal ordering expressions of creation and annihilation operators (see $[12,13])$.

\section{Further Generalization}

The $r$-Whitney numbers of the second kind [17], denoted by $W_{m, r}(n, k)$, are certain extension of noncentral Stirling numbers. In particular, $S_{a}(n, k)=W_{1,-a}(n, k)$. Properties of noncentral Stirling numbers of the second kind can be deduced from those of $r$-Whitney numbers of the second kind by taking $m=1$ and $r=-a$. One may see [17] for a more detailed discussion of $r$-Whitney numbers of the second kind.

The Dowling numbers, denoted by $D_{m}(n)$, were defined as the sum of Whitney numbers of the second kind (see $[2,3]$ ). Hence, one may define the $r$-Dowling numbers, say denoted by $D_{m, r}(n)$, as

$$
D_{m, r}(n)=\sum_{k=0}^{n} W_{m, r}(n, k) \text {. }
$$

These numbers are equivalent to $(r, \beta)$-Bell numbers [7] and they are certain extension of non-central Bell numbers. In fact, $B_{a}(n)=D_{1,-a}(n)$.

On the other hand, the $(r, \beta)$-Bell numbers, denoted by $G_{n, \beta, r}$, were shown to have the following Hankel transform [7]

$$
\operatorname{det}\left(G_{i+j, \beta, r}\right)_{0 \leq i, j \leq n}=\prod_{k=0}^{n} \beta^{k} k !=\beta^{\left(\begin{array}{c}
n+1 \\
2
\end{array}\right)} n ! ! .
$$

This Hankel transform has been shown using the same method employed to obtain the above alternative solution 
for the Hankel transform of $B_{a}(n)$. In this section, we are going to give an alternative proof for (15) following the method in doing the first proof for the Hankel transform of $B_{a}(n)$.

Let $M=\left(a_{n, k}\right)$ be the infinite lower triangular matrix defined recursively by,

$$
\begin{aligned}
a_{n, k}= & a_{n-1, k-1}+(\beta k+r+1) a_{n-1, k} \\
& +\beta(k+1) a_{n-1, k+1}
\end{aligned}
$$

where $n \geq 1, a_{0,0}=1, a_{0, k}=0$ if $k>0$, and $a_{n, k}=0$ if $n<k$. Then we have the following lemma.

Lemma 4.1. Let $\Phi_{k}(y)$ be the exponential generating function of the kth column of matrix $M$, that is,

$$
\Phi_{k}(y)=\sum_{n=0}^{\infty} a_{n, k} \frac{y^{n}}{n !}
$$

Then

$$
\Phi_{k}(y)=e^{\beta-1\left(e^{\beta y}-1\right)+r y} \cdot \frac{\left(e^{\beta y}-1\right)^{k}}{\beta^{k} k !},
$$

where $k \geq 0$ and $\Phi_{0}(y)=\sum_{n=0}^{\infty} G_{y, r, \beta} \frac{y^{n}}{n !}$. That is, the 0column entries of $M$ are $G_{y, r, \beta}, n=0,1,2, \ldots$.

Proof. Using the recurrence relation in (16), we obtain

$$
\begin{aligned}
& \sum_{n=0}^{\infty} a_{n, k} \frac{y^{n-1}}{(n-1) !} \\
= & \sum_{n=0}^{\infty} a_{n-1, k-1} \frac{y^{n-1}}{(n-1) !}+(\beta k+r+1) \sum_{n=0}^{\infty} a_{n-1, k} \frac{y^{n-1}}{(n-1) !} \\
& +\beta(k+1) \sum_{n=0}^{\infty} a_{n-1, k+1} \frac{y^{n-1}}{(n-1) !} .
\end{aligned}
$$

This implies that

$$
\begin{aligned}
\Phi_{k}^{\prime}(y)= & \Phi_{k-1}(y)+(\beta k+r+1) \Phi_{k}(y) \\
& +\beta(k+1) \Phi_{k+1}(y) .
\end{aligned}
$$

With $\Phi_{k}(y)=\frac{e^{v}\left(e^{\beta y}-1\right)^{k}}{\beta^{k} k !}, v=\beta^{-1}\left(e^{\beta y}-1\right)+r y$, the left-hand side of (18) yields

$$
\begin{aligned}
\Phi_{k}^{\prime}(y)= & e^{\beta^{-1}\left(e^{\beta y}-1\right)+r y} \cdot \frac{\left(e^{\beta y}-1\right)^{k-1}}{\beta^{k}(k-1) !} \cdot \beta e^{\beta y} \\
& +\left(\beta \beta^{-1} e^{\beta y}+1\right) e^{\beta-1\left(e^{\beta y}-1\right)+r y} \cdot \frac{\left(e^{\beta y}-1\right)^{k}}{\beta^{k} k !} \\
= & e^{\beta^{-1}\left(e^{\beta y}-1\right)+r y} \cdot \frac{\left(e^{\beta y}-1\right)^{k-1}}{\beta^{k-1}(k-1) !} \cdot e^{\beta y} \\
& +e^{\beta^{-1}\left(e^{\beta y}-1\right)+r y} \cdot \frac{\left(e^{\beta y}-1\right)^{k}}{\beta^{k} k !} \cdot e^{\beta y} \\
& +e^{\beta^{-1}\left(e^{\beta y}-1\right)+r y} \cdot \frac{\left(e^{\beta y}-1\right)^{k}}{\beta^{k} k !} \cdot r
\end{aligned}
$$

While the right-hand side of (18) gives

$$
\begin{aligned}
& \Phi_{k-1}(y)+(\beta k+r+1) \Phi_{k}(y)+\beta(k+1) \Phi_{k+1}(y) \\
& =\frac{e^{v}\left(e^{\beta y}-1\right)^{k-1}}{\beta^{k-1}(k-1) !}+(\beta k+r+1) \frac{e^{v}\left(e^{\beta y}-1\right)^{k}}{\beta^{k} k !} \\
& +\beta(k+1) \frac{e^{v}\left(e^{\beta y}-1\right)^{k+1}}{\beta^{k+1}(k+1) !} \\
& =\frac{e^{v}\left(e^{\beta y}-1\right)^{k-1}}{\beta^{k-1}(k-1) !}+\frac{e^{v}\left(e^{\beta y}-1\right)^{k}}{\beta^{k-1}(k-1) !}+\frac{e^{v}\left(e^{\beta y}-1\right)^{k}}{\beta^{k} k !} \\
& +\frac{e^{v}\left(e^{\beta y}-1\right)^{k}}{\beta^{k} k !} \cdot r+\frac{e^{v}\left(e^{\beta y}-1\right)^{k}\left(e^{\beta y}-1\right)}{\beta^{k} k !} \\
& =\frac{e^{v}\left(e^{\beta y}-1\right)^{k-1}}{\beta^{k-1}(k-1) !}+\frac{e^{v}\left(e^{\beta y}-1\right)^{k-1}\left(e^{\beta y}-1\right)}{\beta^{k}(k-1) !} \\
& +\frac{e^{v}\left(e^{\beta y}-1\right)^{k}}{\beta^{k} k !} \cdot r+\frac{e^{v}\left(e^{\beta y}-1\right)^{k} e^{\beta y}}{\beta^{k} k !} \\
& +\frac{e^{v}\left(e^{\beta y}-1\right)^{k}}{\beta^{k} k !}-\frac{e^{v}\left(e^{\beta y}-1\right)^{k}}{\beta^{k} k !} \\
& =\frac{e^{v}\left(e^{\beta y}-1\right)^{k-1}}{\beta^{k-1}(k-1) !}-\frac{e^{v}\left(e^{\beta y}-1\right)^{k-1}}{\beta^{k-1}(k-1) !} \\
& +\frac{e^{v}\left(e^{\beta y}-1\right)^{k-1} e^{\beta y}}{\beta^{k-1}(k-1) !}+\frac{e^{v}\left(e^{\beta y}-1\right)^{k}}{\beta^{k} k !} \cdot r \\
& +\frac{e^{v}\left(e^{\beta y}-1\right)^{k} e^{\beta y}}{\beta^{k} k !} \\
& =e^{\beta^{-1}\left(e^{\beta y}-1\right)+r y} \cdot \frac{\left(e^{\beta y}-1\right)^{k-1}}{\beta^{k}(k-1) !} \cdot \beta e^{\beta y} \\
& +\left(\beta \beta^{-1} e^{\beta y}+1\right) e^{\beta^{-1}\left(e^{\beta y}-1\right)+r y} \cdot \frac{\left(e^{\beta y}-1\right)^{k}}{\beta^{k} k !} \\
& =e^{\beta^{-1}\left(e^{\beta y}-1\right)+r y} \cdot \frac{\left(e^{\beta y}-1\right)^{k-1}}{\beta^{k-1}(k-1) !} \cdot e^{\beta y} \\
& +e^{\beta^{-1}\left(e^{\beta y}-1\right)+r y} \cdot \frac{\left(e^{\beta y}-1\right)^{k}}{\beta^{k} k !} \cdot e^{\beta y} \\
& +e^{\beta^{-1}\left(e^{\beta y}-1\right)+r y} \cdot \frac{\left(e^{\beta y}-1\right)^{k}}{\beta^{k} k !} \cdot r
\end{aligned}
$$

which shows that the function $e^{\beta^{-1}\left(e^{\beta y}-1\right)+r y} \cdot \frac{\left(e^{\beta y}-1\right)^{k}}{\beta^{k} k !}$, where $k \geq 0$, is a unique solution to the differential equation (18). Thus, the exponential generating function of the kth column of $M$ is given by

$$
\Phi_{k}(y)=e^{\beta^{-1}\left(e^{\beta y}-1\right)+r y} \cdot \frac{\left(e^{\beta y}-1\right)^{k}}{\beta^{k} k !} .
$$

Lemma 4.2. Let $w_{n}$ be the nth row of $M=\left(a_{n, k}\right)$. Define 


$$
w_{n} \circ w_{m}=\sum_{k \geq 0} a_{n, k} a_{m, k} \beta^{k} k !
$$

Then $w_{n} \circ w_{m}=a_{n+m, 0}=G_{n+m, r, \beta}$ for all $n$ and $m$.

Proof. By induction, if $\mathrm{n}=0$ we have

$$
w_{0} \circ w_{m}=\sum_{k \geq 0} a_{0, k} a_{m, k} \beta^{k} k !
$$

Since $a_{0, k}=0 \forall k>0$,

$$
\begin{aligned}
w_{0} \circ w_{m} & =a_{0,0} a_{m, 0} \beta^{0} 0 !=a_{0,0} a_{m, 0} \\
& =a_{0+m, 0}=G_{n, 0, \beta} \forall m .
\end{aligned}
$$

Suppose that $w_{p} \circ w_{m}=a_{p+m, 0}$ holds for $p \leq n-1$ and all $m$. Then by (16)

$$
\begin{aligned}
& w_{n} \circ w_{m}=\sum_{k \geq 0} a_{0, k} a_{m, k} \beta^{k} k ! \\
& =\sum_{k \geq 0}\left[\begin{array}{c}
a_{n-1, k-1}+(\beta k+r+1) a_{n-1, k} \\
+\beta(k+1) a_{n-1, k+1}
\end{array}\right] a_{m, k} \beta^{k} k ! \\
& =\sum_{k \geq 0} a_{n-1, k-1} a_{m, k} \beta^{k} k !+\sum_{k \geq 0}(\beta k+r+1) a_{n-1, k} a_{m, k} \beta^{k} k ! \\
& \quad+\sum_{k \geq 0} \beta(k+1) a_{n-1, k+1} a_{m, k} \beta^{k} k !
\end{aligned}
$$

By interchanging and reindexing the summation, we have

$$
\begin{aligned}
w_{0} \circ w_{m}= & \sum_{k \geq-1} a_{n-1, k+1-1} a_{m, k+1} \beta^{k+1} k+1 ! \\
& +\sum_{k \geq 0}(\beta k+r+1) a_{n-1, k} a_{m, k} \beta^{k} k ! \\
& +\sum_{k \geq 1} a_{n-1, k} a_{m, k-1} \beta^{k} k ! \\
= & \sum_{k \geq 0} a_{n-1, k} a_{m, k+1} \beta^{k+1}(k+1) ! \\
& +\sum_{k \geq 0}(\beta k+r+1) a_{n-1, k} a_{m, k} \beta^{k} k ! \\
& +\sum_{k \geq 0} a_{n-1, k} a_{m, k-1} \beta^{k} k ! \\
= & \sum_{k \geq 0}\left[\begin{array}{c}
a_{m, k-1}+(\beta k+r+1) a_{m, k} \\
+\beta(k+1) a_{m, k+1}
\end{array}\right] a_{n-1, k} \beta^{k} k !
\end{aligned}
$$

By (16),

$$
w_{0} \circ w_{m}=\sum_{k \geq 0} a_{m+1, k} a_{n-1, k} \beta^{k} k !
$$

By the inductive hypothesis,

$$
w_{0} \circ w_{m}=a_{m+1+n-1,0}=a_{n+m, 0}=G_{n+m, r, \beta} .
$$

Theorem 4.3. The $(r, \beta)$-Bell numbers have the Hankel Transform

$$
\begin{aligned}
& \left|\begin{array}{ccccc}
G_{0, r, \beta} & G_{1, r, \beta} & G_{2, r, \beta} & \ldots & G_{n, r, \beta} \\
G_{1, r, \beta} & G_{2, r, \beta} & G_{3, r, \beta} & \ldots & G_{n+1, r, \beta} \\
\vdots & \vdots & \vdots & \ldots & \vdots \\
G_{n, r, \beta} & G_{n+1, r, \beta} & G_{n+2, r, \beta} & \cdots & G_{2 n, r, \beta}
\end{array}\right| \\
& =\prod_{j=0}^{n} \beta j j !=\beta^{\left(\begin{array}{c}
n+1 \\
2
\end{array}\right)} n ! !
\end{aligned}
$$

where $G_{n, 0,1}=B_{n}$ (Bell numbers) and $G_{n,-\alpha, 1}=B_{\alpha}(n)$ (Non-central Bell numbers).

Proof. Let $M_{n}$ be the lower triangular submatrix of $M$ consisting of the rows and columns numbered 0 to $\mathrm{n}$. Then $M_{n}$ is a matrix with diagonal 1. It follows that $\operatorname{det} M_{n}=1$. This implies that the determinant of upper triangular submatrix $M_{n}^{T}$ is one; that is, $\operatorname{det} M_{n}^{T}=1$. Let $\bar{M}_{n}=\left(\beta^{j} j ! a_{i, j}\right)_{0 \leq i, j \leq n}$. Then

$$
\operatorname{det} \bar{M}_{n}=\prod_{j=0}^{n} \beta j j !
$$

By Theorem 4.2,

$$
\bar{M}_{n} \cdot M_{n}^{T}=\left(b_{i, j}\right)_{0 \leq i, j \leq n}
$$

where $b_{i, j}=\sum_{k=0}^{n} a_{i, k} a_{j, k} \beta j j !=a_{i+j, 0}=G_{i+j, r, \beta}$.

That is,

$$
\bar{M}_{n} \cdot M_{n}^{T}=\left(G_{i+j, r, \beta}\right)_{0 \leq i, j \leq n}
$$

Thus,

$$
\begin{aligned}
\operatorname{det}\left(\bar{M}_{n} \cdot M_{n}^{T}\right) & =\left(\operatorname{det} \bar{M}_{n}\right) \cdot\left(\operatorname{det} M_{n}^{T}\right) \\
& =\prod_{j=0}^{n} \beta^{j} j !=\beta^{\left(\begin{array}{c}
n+1 \\
2
\end{array}\right)} n ! ! .
\end{aligned}
$$

\section{References}

[1] M. Aigner, A Characterization of the Bell Numbers, Discrete Math. 205 (1999), 207-210

[2] M. Benoumhani, On some numbers related to Whitney numbers of Dowling lattices, Advance in Applied Mathematics, 9, pp. 106-116, 1997.

[3] M. Benoumhani, On Whitney Numbers of Dowling Lattices. Discrete Math. pp. 13-33, 1996.

[4] A.Z. Broder, The r-Stirling Numbers, Discrete Math 49(1984), 241-259.

[5] Ch.A. Charalambides and J. Singh, A review of the Stirling numbers, their generalization and statistical applications, Commun Statist.-Theory Meth. 20(8) (1988), 2533-2595.

[6] L. Comtet, Advanced Combinatorics, Reidel, Dordrecht, The Netherlands, 1974.

[7] R.B. Corcino and C.B. Corcino, The Hankel Transform of Generalized Bell Numbers and Its q-Analogue, Utilitas Mathematica, 89 (2012), 297-309.

[8] A. Cvetković, P. Rajković, and M. Ivković, Catalan numbers, The Hankel Transform and Fibonnaci numbers, J. Integer Seq., 5(2002), Article 02.1.3.

[9] M. Desainte-Catherine and X. G. Viennot, Enumeration of certain Young tableaux with bound height, Combinatorie Énumérative (Montreal 1985), Lect. Notes in Math. 1234 (1986), 58-67.

[10] Richard Ehrenborg, The Hankel Determinant of exponential Polynomials, American Mathematical Monthly, 107(2000)557-560.

[11] M. Garcia-Armas and B. A. Seturaman, A note on the Hankel transform of the central binomial coefficients, J. Integer Seq. 11(2008), Article 08.5.8.

[12] Katriel, J., Combinatorial Aspects of Boson Algebra, Lett. Nuovo Cimento 10 (1974) 565-567.

[13] Katriel J. and Kibler M., Normal ordering for deformed boson operators and operator-valued deformed Stirling numbers, J. Phys. A: Math. Gen. 25 (1992), 2683-2691. 
[14] M. Koutras. Non-Central Stirling Numbers and Some Applications. Discrete Math.42 (1982): 73-89.

[15] J.W. Layman, The Hankel transform and some of its properties, $J$ Integer Seq. Vol.4 (2001), Article 01.1.5.

[16] I. Mezö, On the Maximum of r-Stirling Numbers, Adv. in Appl. Math. 41(3) (2008), 293-306.

[17] I. Mezö, A new formula for the Bernoulli polynomials, Result. Math. 58(3) (2010), 329-335.

[18] I. Mezö, The r-Bell numbers, J. Integer Seq. 14 (2011), Article 11.1.1.

[19] C. Radoux, Déterminat de Hankel construit sur des polynomes liés aux nombres de dérangements, European Journal of Combinatorics 12(1991), 327-329.
[20] N. J. Sloane, The On-line Encyclopedia of Integer Sequences, $\mathrm{http}: / / \mathrm{www} \cdot$ research.att.com/njas/sequences.

[21] M.Z. Spivey and L. L. Steil, The k-binomial transform and the Hankel transform, J. Integer Sq. 9(2006), Article 06.1.1.

[22] U. Tamm, Some aspects of Hankel matrices in coding theory and combinatorics, Electron. J. combin. 8(1) A1(2001).

[23] R. Vein and A. Dale, Determinants and Their Applications in Mathematical Physics, Springer, 1991.

[24] Daniel Zelinsky, A First Course in Linear Algebra, 2ed, Academic Press, Inc., 1973. 